\title{
Tyrosine on Cutaneous Pigmentation - A New Therapy Line for Vitiligo
}

\author{
Aruna V*, Soundharya R, Amruthavalli GV and Gayathri R \\ Dr. JRK's Research and Pharmaceuticals Pvt. Ltd, India
}

Submission: October 07, 2020; Published: October 21, 2020

*Corresponding author: Aruna V, Department of Research \& Development, Dr. JRK's Research \& Pharmaceuticals Pvt. Ltd, India

\section{Abstract}

The topical effect of tyrosine in altering skin pigmentogenesis was studied using melanin pigment producing strain of Cryptococcus neoformans. Although the biochemical pathway is different in C. neoformans, where phenol oxidase enzyme is involved unlike tyrosinase as in the case of mammalian cell, still the findings prove the method to be reliable and reproducible. Peri-cellular absorption of tyrosine and subsequent melanogenesis were observed in treated cell of $\mathrm{C}$ neoformans. Further the quality of pigment produced in the cell of the organism was different from phenol oxidase induced pigment. The pigment thus offered protection to the cells from UV damage. The observation based study on tyrosine based cream in human skin and subsequent sun exposure resulted in minimal erythema and delayed pigment darkening indicating the underlying melanogenesis process. Further, the absence of erythema suggests the possible melanogenesis activation at sub-dermal level and its effect in protecting the skin from sun damage. The clinical usefulness of topical tyrosine and other scientific details are discussed in the paper.

Keywords: Vitiligo; Tyrosine; Phenoloxidase; L-Dopa; Guizotia Abyssinica; Uv Treatment; Pigmentogenesis

\section{Introduction}

A rapid, simple, less time consuming, cost effective and finally a reliable and relatable method for screening agents/ingredients and developing a formulation for the management of vitiligo and hypopigmentary disorders of the skin is greatly lacking [1]. Although cell culture methods are available and are used widely for the above purpose, but still the extrapolation of the cell culture findings to predict the possible treatment outcome based on the cell culture assay of agents/ingredients is quite tricky, confusing and in most cases, not relatable [2]. Several microorganisms are known to produce melanin like substance for their defense and the fungi -Cryptococcus neoformans is the best example for melanin synthesis [3]. In general, the microbes synthesize melanin mostly via phenoloxidase but in mammalian system, tyrosinase replaces the role of phenoloxidase. In any case L-DOPA and tyrosine are the precursor molecules for the biosynthesis of melanin [4]. The extracellular supplementation of tyrosine into the growth medium of C.neoformans was found to accelerate the biochemical process of melanogenesis. Further the melanin thus formed was found to accumulate in the cells but a distinct capsule formation around the cell was absent. C. neoformans being a 'simple life unit'; any changes in the cells of C.neoformans therefore can be easily corelated to the mammalian system [5].

In the case of cell culture assay, where the melanocytes are used but the melanocytes always exist only as an individual cell unit and may not reflect the possible response of the skin in to [6]. Therefore the study outcome that is obtained with cryptococcal cells is more relatable to the cutaneous response than the use of melanocytes. In our present study we have established the extracellular tyrosine induced melanogenesis in cryptococcal cells and such cells are relatively protected from UV induced cell death/mutation. The interesting feature was that the cells grown in tyrosine supplemented media showed greater resistance to UV treatment than the cells grown in guizotia abyssinica agar supplemented with creatinine. Vitiligo being an autoimmune disorder with progression tendency, palliative, tyrosine based therapy may be effective for long term purposes even if the treatment outcome is relatively sluggish $[7,8]$. In the case of progressive vitiligo, tyrosine based treatment even if it retards the progression of hypopigmentation by $50 \%$ still such treatment would be highly valuable. In the present study we report the use of 
extracellular supplementation of tyrosine and its usefulness in the treatment of vitiligo. Details are presented in the paper.

\section{Materials and Methods}

\section{Growth of C.neoformans in tyrosine supplemented media}

Sabouraud's dextrose agar supplemented with 3 different concentrations of tyrosine such as $0.1,0.2,0.3 \mu \mathrm{g} / \mathrm{ml}$ respectively were used for the experiment. The freshly grown C.neoformans were inoculated and incubated in the above media condition for 7 days and then observed the colony and the cells for melanin, both macroscopically and microscopically $[9,10]$.

\section{Growth of C.neoformans in guizotia abyssinica agar with creatinine}

Cryptococcal cells were grown in guizotia abyssinica agar supplemented with creatinine and incubated for 7 days. After 7 days the colony characteristics and cells were observed for melanin as described above $[11,12]$.

\section{Response of cells to UV}

C.neoformans grown in tyrosine supplemented media and from guizotia abyssinica agar supplemented with creatinine were grown again in sabouraud's agar and were then immediately were exposed to UV for 5 minutes. The number of colonies grown was counted and the melanin synthesized in the cells vis-à-vis UV protection was ascertained.

\section{Melanin quantification}

Melanin produced in C.neoformans cells grown in tyrosine supplemented media and guizotia abyssinica agar supplemented with creatinine was quantified using a standard procedure. Approximately $10^{9}$ cells grown in different experimental setup were pelleted by centrifugation at $1,000 \mathrm{~g}$ for $5 \mathrm{~min}$ and then the cells were washed with phosphate-buffered saline. After re- centrifugation as described above, the supernatant was decanted, the cells were precipitated again, re-suspended in distilled water and $1 \mathrm{ml}$ of ethanol - ether 1:1 ( $\mathrm{vol} / \mathrm{vol})$ was added to remove the artifact other than melanin. Cell wall lysis procedure was also followed. The insoluble melanin in ethanol-ether was resuspended and centrifuged at 3,000 g for $5 \mathrm{~min}$, the precipitate was dissolved in $1 \mathrm{ml}$ of $1 \mathrm{~N} \mathrm{NaOH} / 10 \%$ dimethyl sulfoxide at 8 ${ }^{\circ} \mathrm{C}$ for $30 \mathrm{~min}$. The absorbance was measured at $470 \mathrm{~nm}$, and the melanin synthesized in treated and control cells were calculated.

Effect of topical tyrosine on melanogenesis and erythema formation in human volunteer

Five human volunteer were chosen for the study. The cream with tyrosine $(0.2 \%)$ and without tyrosine was applied over the volar forearm region at $2 \mathrm{mg} / \mathrm{cm}^{2}$ separately. Then the volar forearm region was exposed to sunlight for 15 minutes and the extent of erythema formed in the treated and control sites and between products with and without tyrosine was recorded immediately after exposure and the pigment production (tanning) in the above experimental set up also recorded after 24 hours $[13,14]$.

\section{Result}

\section{Growth of C.neoformans in tyrosine supplemented media}

The cells grown in tyrosine supplemented media showed the melanin synthesis which is directly proportional to the concentration of tyrosine used (Figure 1-4). C.neoformans grown in guizotia abyssinica agar supplemented with creatinine did produce intense pigmentation. Microscopic appearance of C.neoformans cells grown in tyrosine supplemented media showed the accumulation of melanin to be higher in cells grown in tyrosine supplemented media with cell wall distinctively with devoid of capsule formation and cell enlargement (Figure 5-8).

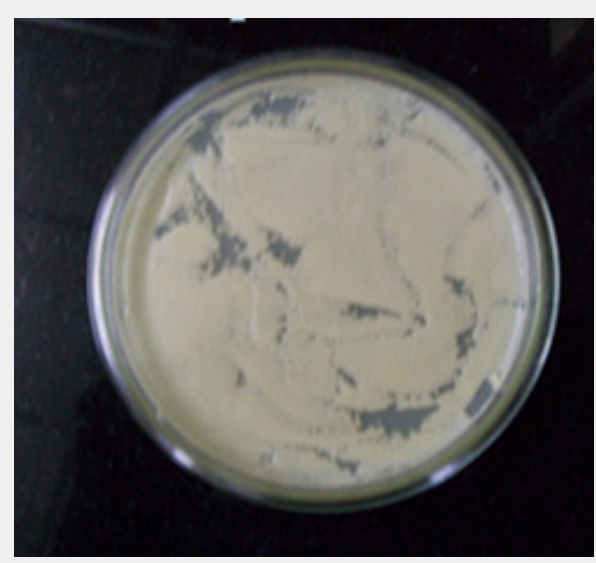

Figure 1: C.neo in SDA. 


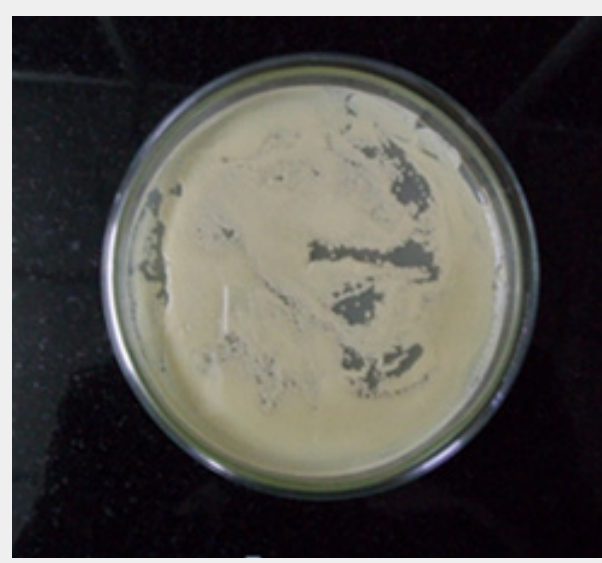

Figure 2: C.neo in SDA with $0.1 \mu \mathrm{g} / \mathrm{ml}$ tyrosine.

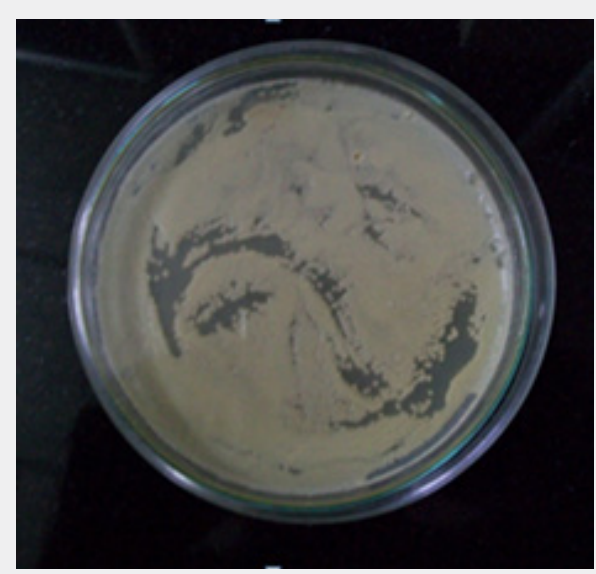

Figure 3: C.neo in SDA with $0.2 \mu \mathrm{g} / \mathrm{ml}$ tyrosine.

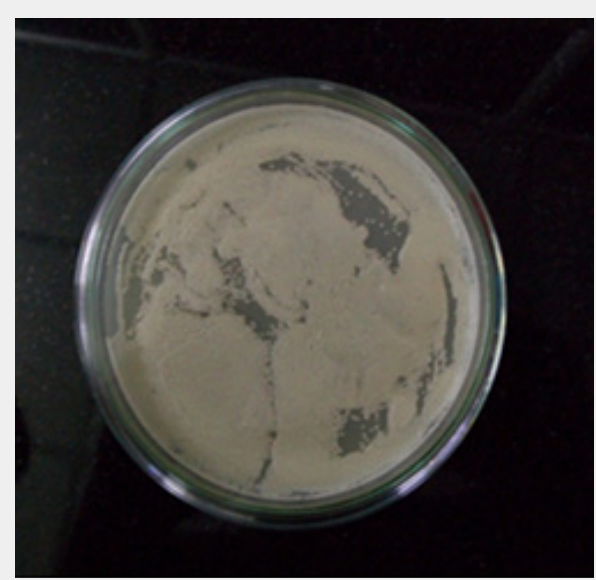

Figure 4: C.neo in SDA with $0.3 \mu \mathrm{g} / \mathrm{ml}$ tyrosine.

\section{Response of cells to UV}

We have used 500 CFU's of C.neoformans for UV exposure. UV exposure in the case of the organism grown in SDA showed a significant reduction in the colonies. Only 13/500 could grow after UV exposure. Whereas the UV untreated plate yielded 496/500
CFU's. In the case of organism grown in tyrosine supplemented media when exposed to UV, 76\% CFU's could withstand the UV treatment with only $7.8 \%$ of the cells that were grown were mutant colonies. In the case of organism grown in Guizotia abyssinica agar supplemented with creatinine media when exposed to UV yielded only $24 \%$ of cells with $93.3 \%$ of colonies were mutant (Table 1 ). 
Figure 5: Control.
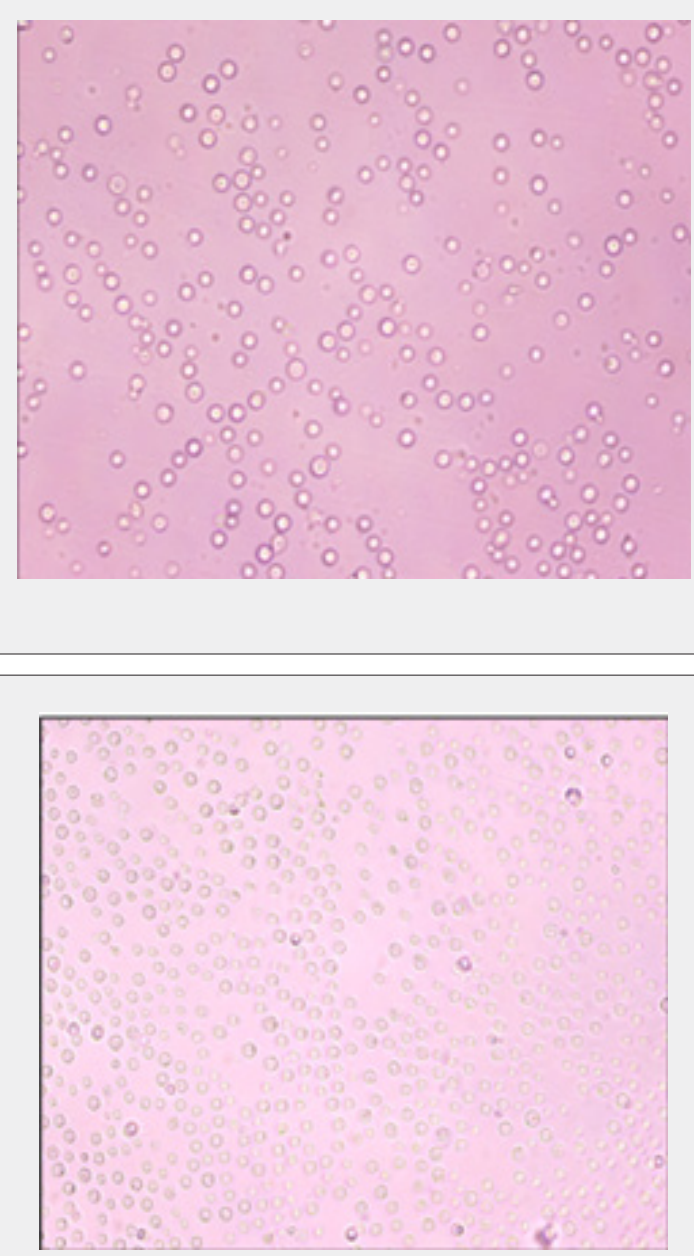

Figure 6: $0.1 \mu \mathrm{g} / \mathrm{ml}$ tyrosine.

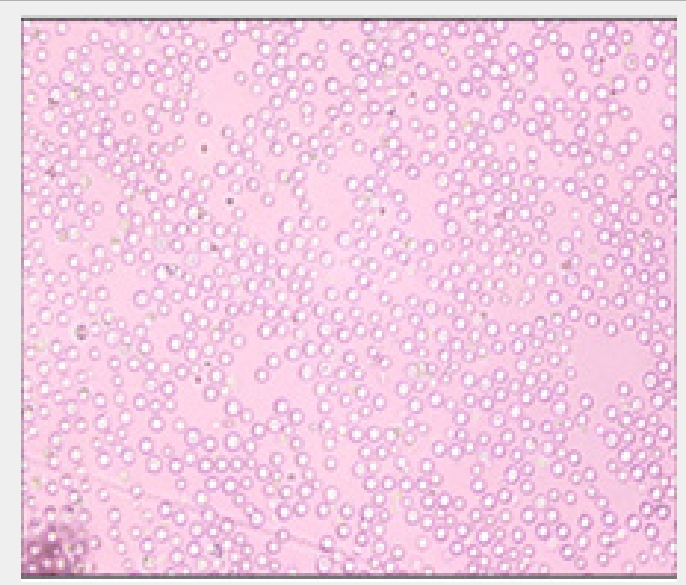

Figure 7: $0.2 \mu \mathrm{g} / \mathrm{ml}$ tyrosine.

\section{Melanin quantification}

Tyrosine treatment significantly increased the melanogenesis in a concentration dependent manner. The C.neoformans grown in guizotia abyssinica agar supplemented with creatinine also showed melanin synthesis (Table 2).
Effect of topical tyrosine on melanogenesis and erythema formation in human volunteer

None of the 5 volunteers treated with tyrosine incorporated cream showed erythema response to sun exposure whereas all of them showed varying degrees of tanning after 24 hours (Table 3). 


\section{Juniper Online Journal of Dermatology \& Cosmetics}

None of the 5 volunteers treated with the cream devoid of tyrosine showed any tanning response after 24 hours. However all of them

showed varying degrees of erythema within 15 minutes of sun exposure (Table 4).

Figure 8: $0.3 \mu \mathrm{g} / \mathrm{ml}$ tyrosine.

Table 1: No of CFU's and mutant colonies after exposure to UV in different treatment conditions.

\begin{tabular}{|c|c|c|}
\hline Treatment & CFU & Mutant cells \\
\hline Grown in SDA (no UV exposure) & $496 / 500(99.2 \%)$ & - \\
\hline Grown in SDA (After UV exposure) & $13 / 500(2.6 \%)$ & $13 / 500(2.6 \%)$ \\
\hline Grown in tyrosine media (after UV exposure) & $380 / 500(76 \%)$ & $30 / 380(7.8 \%)$ \\
\hline Grown in Guizotia abyssinica agar (after UV exposure) & $120 / 500(24 \%)$ & $112 / 120(93.3 \%)$ \\
\hline
\end{tabular}

Table 2: C.neoformans grown in guizotia abyssinica agar supplemented with creatinine also showed melanin synthesis.

\begin{tabular}{|c|c|}
\hline Treatment & \% of melanin synthesized \\
\hline SDA & 16 \\
\hline \multicolumn{2}{|c|}{ Tyrosine supplemented media } \\
\hline 0.1 & 21 \\
\hline 0.2 & 34 \\
\hline 0.3 & 46 \\
\hline Guizotia abyssinica agar & 52 \\
\hline
\end{tabular}

Table 3: Erythema and tanning response in volunteers treated with tyrosine incorporated cream.

\begin{tabular}{|c|c|c|c|c|}
\hline \multirow{2}{*}{ Volunteers } & \multicolumn{3}{|c|}{ Dermal changes after exposure to sun for 15 minutes } \\
\cline { 2 - 5 } & \multicolumn{2}{|c|}{ Erythema (within 15 minutes) } & \multicolumn{2}{c|}{ Tanning (after 24 hours) } \\
\cline { 2 - 5 } & Control & Treated & - & + \\
\hline 1 & - & - & - & ++ \\
\hline 2 & - & - & - & ++ \\
\hline 3 & - & - & - & ++ \\
\hline 4 & - & - & - & + \\
\hline 5 & - & - & & ++ \\
\hline
\end{tabular}

Table 4: Erythema and tanning response in volunteers treated with cream devoid of tyrosine.

\begin{tabular}{|c|c|c|c|c|}
\hline \multirow{2}{*}{ Volunteers } & \multicolumn{4}{|c|}{ Dermal changes after exposure to sun for 15 minutes } \\
\cline { 2 - 5 } & \multicolumn{2}{|c|}{ Erythema (within 15 minutes) } & \multicolumn{2}{c|}{ Tanning (after 24 hours) } \\
\cline { 2 - 5 } & Control & Treated & - & Treated \\
\hline 1 & - & +++ & - & - \\
\hline 2 & - & + & - & - \\
\hline 3 & - & ++ & - & + \\
\hline 4 & - & +++ & - & - \\
\hline
\end{tabular}




\section{Discussion}

Our present investigation has brought out a new treatment possibility for vitiligo and hypopigmentary disorders of the skin. Tyrosine is although known as the fundamental unit of skin pigment called melanin but still tyrosine is not the sort after treatment option for vitiligo. It is always assumed that amelanotic condition or the ability of the melanocytes to produce the pigment or due to the enzymatic or the biochemical pathway aberrations as the causes for vitiligo. Substrate availability in excess may sometime offer solution to the problem. However which patient may require such treatment option is difficult to predict because the etiopathology of vitiligo and hypopigmentary disorders of the skin is too complex. Therefore simultaneously adopting several treatment options to address various pathological triggers and correcting each of them is necessary. In the present study, we have explored how the extracellular supplementation of tyrosine would increase pigmentogenesis in C.neoformans. In our earlier study we have already established the use of C.neoformans in the screening and identifying the skin pigment modulators as effective as melanocytes based screening methodology.

The advantage of using C.neoformans is that the C.neoformans is a eukaryotic microorganism and also it represents the life in totality. Whereas, the melanocytes are one of the cells in the skin and it requires several direct and indirect communication network with other cells and the feedback mechanism to function. Therefore the scope of predicting the therapeutic usefulness of any compound or agent tested by C.neoformans will have a higher relatability than melanocyte or tyrosinase enzyme based screening outcome. The C.neoformans cells treated with varying concentration of tyrosine has significantly increased the melanogenesis, melanin accumulation and cell wall conspicuous. Further the pigmentogenesis in C.neoformans had occurred in 7 days as against 14 days in the case of guizotia abyssinica agar supplemented with creatinine. Interestingly the capsule formation was fairly less in C.neoformans cells grown in tyrosine supplemented media than guizotia abyssinica agar supplemented with creatinine. The formation of capsule is linked more with virulence and the melanoid formation is linked with the survival of cells.

The pigmentogenesis with poor capsule formation therefore suggests that the tyrosine may be serving only as substrate for the pigmentation and other than that the tyrosine may not alter the virulence mechanism of organism. The absorption of tyrosine from the media by C.neoformans cells clearly suggests the possibility of dermal absorption of tyrosine because the cell wall architecture of C.neoformans is quite impermeable for the easy entry of agents. Therefore a cream with tyrosine may offer better relief to the problems of hypopigmentary disorders; where the substrate abundance is sufficient to promote pigmentation. The microscopic examination of the cells clearly reiterates that tyrosine induces melanogenesis in a dose dependent manner with not much alteration either in the cell size or in the cell wall armamentarium. The cells grown in different growth conditions such as SDA, tyrosine supplementation and guizotia asyssinica agar were exposed to UV to check how the melanogenesis in the cells offer protection from UV.

The cells grown in SDA could not withstand UV exposure and hence majority of the cells could not survive and only a limited number of mutant colonies alone could survive. In the case of tyrosine treatment about $70 \%$ of the cells survived UV exposure clearly suggesting that the melanin produced by the cells may be responsible for the UV protection. The countercheck measures on mutant colonies are also revealed only the above possibility. Whereas the cells grown in guizotia abyssinica agar supplemented with creatinine could not withstand UV exposure despite high melanin synthesis. We presume that the phenol shock in the guizotia abyssinica agar may have made the cells vulnerable to UV further the melanin synthesis in the cells in the above media may not be uniform and hence the survival rate of cells vis-àvis mutant formation were almost equal. The quantification of melanin assay has revealed that C.neoformans grown in tyrosine supplemented media yielded pigment which is slightly less blackish than the C.neoformans cells grown in guizotia abyssinica agar supplemented with creatinine.

C.neoformans produces pigment through a different biochemical pathway than the mammalian cells. Phenol oxidase enzyme in C.neoformans which is an analogue of tyrosinase in a mammalian system results in pigment production in C.neoformans. The reason for the poor survival rate of C.neoformans when exposed to UV despite melanin synthesis may be due to the phenol shock in guizotia abyssinica agar medium. The qualitative aspect of the pigment produced by the cells grown in tyrosine supplemented media was significantly different from that of the cells grown in guizotia abyssinica agar supplemented with creatinine; wherein the melanin was densely colored may be due to the capsule inclusion. We presume that the tyrosine may trigger melanogenesis although through phenoloxidase pathway but may be limiting the events just to pigmentogenesis. Whereas the cells grown in guizotia abyssinica agar supplemented with creatinine may pose 'phenol threat' to the cells and hence the production of pigment, capsule formation and other associated changes may be taking place in the cells and hence the resultant product is quite dark and dense but still not protecting the cells from UV exposure. Subsequent to the above experiment, we also tried to answer the question of whether topical tyrosine when results in pigmentogenesis also alter the inflammatory responses.

We have used two sets of creams viz., one with tyrosine and without tyrosine. The solar effect on tyrosine supplemented and the cream devoid of tyrosine was checked by applying the creams over the volar forearm region in volunteers and then expose to sunlight. To our surprise we found that tyrosine supplemented cream did not cause erythema formation in response to sun 
exposure, however resulted in delayed pigment darkening (tanning) after 24 hours. Tyrosine doesn't filter down UV A selectively and hence the delayed pigment darkening necessarily cue only melanogenesis due to abundant substrate availability. The sun exposure has triggered the evolutionary instinct of the skin to produce pigment and when the substrate was available the pigmentogenesis has taken place. The process involved in melanin pigmentation might have protected the skin and hence no erythema formation was observed in tyrosine supplemented cream was when used.

The above findings further suggest that tyrosine may not only augur melanogenesis in the skin but also may permeate through skin when applied topically. However the delayed pigment darkening in volar forearm region treated with tyrosine supplemented cream disappeared by $48 \mathrm{hrs}$ clearly indicating the melanogenesis event was only responsible for tanning and not the dermal protective response to sun exposure. The cream devoid of tyrosine did not offer sun protection and hence the erythema was obvious within 15 minutes of sun exposure. But no immediate or delayed pigment darkening has occurred. The characterization of melanin by Electron Paramagnetic Resonance spectra (EPR) data not included showed that the pigment produced by C.neoformans cells grown in tyrosine supplemented media was distinctly different from that of guizotia abyssinica agar supplemented with creatinine. Topical tyrosine may offer the much needed therapeutic benefit to vitiligo patients as revealed by our study.

However such positive response is possible only in those patients who may suffer from the scarcity of the substrate of melanin- Tyrosine. Differentiating the different etiological causes of vitiligo and accordingly modifying the treatment may not be possible in the case of vitiligo and hence all treatment options such as increased substrate availability, promotion of tyrosinase activity, increased dendrite number and length in melanocytes and accelerating the melanin transfer mechanism to keratinocytes are required. Immunosuppressive agents also required if the active predation of melanocytes by the self -immune defense mechanism were the cause for the pigment loss. Our study has brought out a ray of hope for the treatment of vitiligo. Considering the tyrosine being the last biochemical molecule (amino acid) with decent bio-absorption through skin tyrosine based treatment option may hold key to vitiligo treatment in near future. The cream partitioning co-efficient is necessary for the rapid and sustained release of tyrosine from the cream and therefore it is not just the use of tyrosine alone may offer the result but the formulation science also plays a significant role.

\section{References}

1. Hossani MA, H Rebat (2011) Treatment of vitiligo: Advantages and disadvantages, indications for use and outcomes. Giornale italiano di dermatologia e venereologia : organo ufficiale, Società italiana di dermatologia e sifilografia 146(5): 373-395.

2. Dell'Anna ML, Cario M, Bellei B, Taieb A, Picardo M (2012) In vitro research on vitiligo: Strategies, principles, methodological options and common pitfalls. Experimental dermatology 21(7): 490-496.

3. Mcfadden DC, Casadevall A (2001) Capsule and melanin synthesis in Cryptococcus neoformans. Medical Mycology 39 (Supp 1): 19-30.

4. Plonka P, Grabacka M (2006) Melanin synthesis in microorganisms Biotechnological and medical aspects. Acta biochimica Polonica 53(3): 429-433.

5. Ellis DH, Pfeiffer TJ (1990) Natural habitat of Cryptococcus neoformans var. gattii. Journal of clinical microbiology 28(7): 1642-1644.

6. Gill BS, Brar MS, Chaudhary N, Randhawa A (2019) Non-cultured melanocyte transfer in the management of stable vitiligo. J Family Med Prim Care 8(9): 2912-2916.

7. Namazi MR, Chee Leok GO (2009) Vitiligo and diet: a theoretical molecular approach with practical implications. Indian J Dermatol Venereol Leprol 75(2): 116-118.

8. Gianfaldoni S, Tchernev G, Lotti J, Uwe W, Satolli F, et al. (2018) Unconventional Treatments for Vitiligo: Are They (Un) Satisfactory? Open Access Maced J Med Sci 6(1): 170-175.

9. Chaskes S, Tyndall RL (1975) Pigment production by Cryptococcus neoformans from para- and ortho-diphenols: effect of the nitrogen source. J Clin Microbiol 1(6): 509-514.

10. Nurudeen TA, Ahearn DG (1979) Regulation of melanin production by Cryptococcus neoformans. J Clin Microbiol 10 (5): 724-729.

11. Paliwal DK, Randhawa HS (1978) Evaluation of a simplified Guizotia abyssinica seed medium for differentiation of Cryptococcus neoformans. J. Clin. Microbiol 7(4): 346-348.

12. Staib F (1962) Cryptococcus neoformans and Guizotia abyssinica (syn. G. oleifera D.C.) (farbreadktion für C. neoformans). Z Hyg Infektionskr 48(5): 1481962466475.

13. Satarupa M, Aruna V, Gayathri R (2017) Villainous Effect of Sun and Goodness of Evefresh. Skin Dis Skin Care. 2:20

14. Aruna V, Gayathri R (2015) Complimentary benefits from contradicting agents - Science of a novel cream for melanogenesis. Journal of Applied Cosmetology 33(3): 123-128. 


\section{Your next submission with Juniper Publishers} will reach you the below assets

- Quality Editorial service

- Swift Peer Review

- Reprints availability

- E-prints Service

- Manuscript Podcast for convenient understanding

- Global attainment for your research

- Manuscript accessibility in different formats

( Pdf, E-pub, Full Text, Audio)

- Unceasing customer service

Track the below URL for one-step submission https://juniperpublishers.com/online-submission.php 